\title{
Magnetic Resonance Angiography
}

\section{OVERVIEW}

The "flow compensation" of moving spins, as described in UNIT B7.2, is necessary to visualize blood in MR imaging. In this unit, we consider the effects of blood flow in more detail, particularly in the desire for an accurate measurement of the blood vessel lumen (the interior dimensions of the vessel). The imaging of blood vessels (arteries and veins) is often referred to as magnetic resonance angiography (MRA).

We have seen that the presence of motion can corrupt the MR signal in a number of ways. It can cause blurring, ghosting and loss of signal. However, with a careful design of the MR sequence it was shown that the signal from moving spins can be made to appear as if they were not moving at all, and that there will be good signal present in the blood itself. This suggests the potential of using MR to actually obtain an image of the blood vessels. Ideally, the signal from the blood could be made so bright that, with the appropriate processing, a complete angiogram of the vessels could be obtained. In this unit, we review the concepts behind time-of-flight (TOF) MRA.

We begin by discussing two different approaches, 2-D and 3-D MRA. Our imaging goal is to have a bright signal from blood vessels. Thus, the 3-D MRA is more useful for fast flow and the 2-D MRA for both slow flow or fast flow. Two-dimensional MRA is used to image slow flow for a very simple reason. When the blood is excited by an RF pulse and moves too slowly, for a fixed slice thickness and for short $T_{\mathrm{R}}$ experiments the signal gets saturated, i.e., it is suppressed. This is because the longitudinal magnetization has had little chance to recover from one RF pulse to the next. But for flowing spins, say those moving perpendicular to the slice, if they have a speed $v$ greater than $\mathrm{TH} / T_{\mathrm{R}}$, then all the blood that was excited for one RF pulse will have left the imaging plane and new blood will enter. This new blood has never seen an RF pulse before, so its signal will not be saturated (Fig. B7.3.1). Clearly, for small TH, this is usually the case. Hence, we have the marvelous situation whereby the signal from blood remains bright and that from the background gets suppressed (see panel A of Fig. B7.3.17). For example, for a velocity of $10 \mathrm{~cm} / \mathrm{sec}$ and a repeat time of $20 \mathrm{msec}$, the blood will have moved $2 \mathrm{~mm}$ in one slice. A choice of a $2 \mathrm{~mm}$ slice thickness then allows complete refreshment of the blood. In practice, even for the slower flow of $5 \mathrm{~cm} / \mathrm{sec}$, it only takes two RF pulse cycles for the blood to be refreshed so a smaller flip angle $\left(30^{\circ}\right.$ to $\left.60^{\circ}\right)$ would still do an excellent job highlighting the blood and suppressing the background tissue.

For 3-D imaging, the situation is not so simple. For spins flowing through the plane of the 3-D slab, those near the front end will yield a bright signal for the early slices while those that arrive near the end of the slab after many RF pulses will have been suppressed in a manner similar to the stationary tissue (see Fig. B7.3.3 and panels A and B of Fig. B7.3.6). This is not a serious problem if $v T_{R}$ is roughly equal to the slab thickness. So one solution when imaging the brain, for example, where the speed of the blood is $\sim 50 \mathrm{~cm} / \mathrm{sec}$, is to reduce the slab thickness to $\sim 2$ to $3 \mathrm{~cm}$ and do a multi-slab 3-D acquisition. A second approach is to suppress the background signal even further using a magnetization transfer pulse (see panels A and B of Fig. B7.3.8). A third approach is to vary the flip angle spatially so the spins entering the slab see a smaller flip angle and those further downstream see a larger flip angle. This spatially variable flip angle approach makes it possible to make the blood signal more uniform throughout the slab (Fig. B7.3.7). A fourth, more robust, approach is to use a contrast agent to reduce the $T_{1}$ of the blood. If the $T_{1}$ of the blood is reduced to $\sim 5$ times the $T_{\mathrm{R}}$ value or less, the effects of saturation are dramatically reduced

Contributed by Yu-Chung Norman Cheng and E. Mark Haacke

Current Protocols in Magnetic Resonance Imaging (2002) B7.3.1-B7.3.18

Copyright (@) 2002 by John Wiley \& Sons, Inc.
Motion Artifacts, Motion Compensation, and Magnetic Resonance Angiography

\section{B7.3.1}

Supplement 7 
because the signal has had a chance to regrow much more quickly between RF pulses (Fig. B7.3.11).

Practically, it is also very useful to suppress the veins when the arteries are the main interest. This can be accomplished by applying another RF pulse in an appropriate location to suppress the veins. For example, when imaging the carotid arteries, a cranially placed saturation pulse will suppress the signal from the jugular veins (Fig. B7.3.17).

The display of a large series of slices to highlight the vessels is accomplished by a maximum intensity projection method. Simply put, if one looks through a stack of slices along a given line of sight and choose the maximum signal seen at a given pixel, the resulting image is referred to as an MIP of the stack. When an MIP is created from a 3-D data set one can look at different angles to make new projections. This makes it easier to interpret the images when there are lots of vessels or overlapping vessels.

\section{TECHNICAL DISCUSSION}

\section{Inflow or Time-of-Flight (TOF) Effects}

Most MRA methods in use today use short $T_{\mathrm{R}}$ or gradient echo imaging methods. Our focus in this unit is on taking advantage of the spins' motion to create MR angiograms that are easily understood and interpreted.

\section{Critical speeds}

Consider plug flow with speed $v$ in a cylinder (Fig. B7.3.1). If region A with slice (slab) thickness $T H$ is excited with the first RF pulse of a 2-D (3-D) gradient echo experiment, then a time $T_{R}$ later, just before excitation by the second RF pulse, the blood in region $\mathrm{C}$, with the same thickness TH will have completely replaced that in the slice if $z_{2}=v T_{\mathrm{R}}>$ $\mathrm{TH}$, where $z_{2}$ is the distance shown in the figure. In general, there is complete refreshment when $v T_{R}>T H$, or:

$$
v>v_{c}
$$

where:
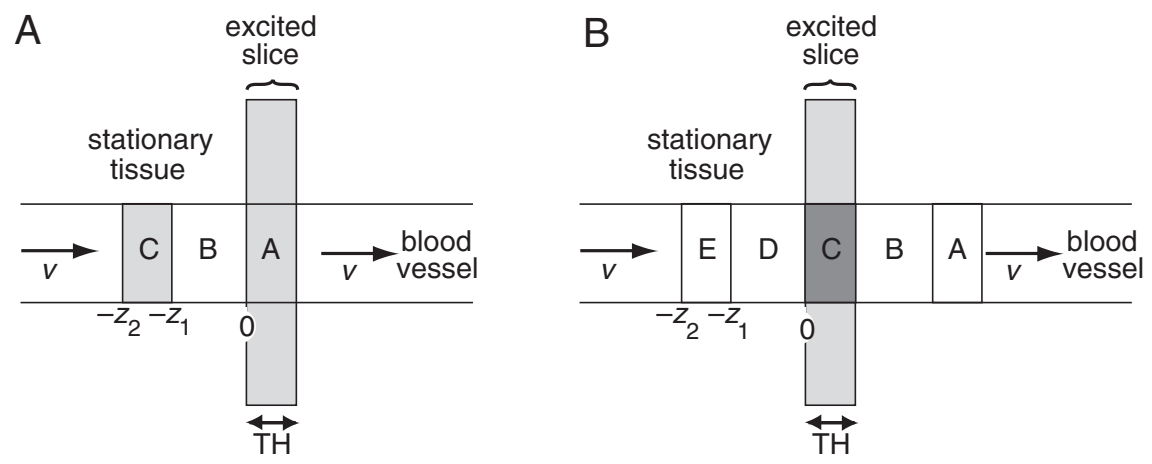

Figure B7.3.1 Illustration for explaining TOF in the presence of plug flow with speed $v$ in the $z$ direction. (A) For TH less than $v T_{R}$, spins in the blood in region A see one RF pulse and then exit the slice before it is excited again. The spins in region $C\left(T H=z_{2}-z_{1}, z_{i}>0\right)$ are not affected by the first RF pulse but (B) see the second one when they enter the slice and occupy the $z$-region from 0 to $\mathrm{TH}$. 


$$
v_{c}=\mathrm{TH} / T_{\mathrm{R}}
$$

To belabor it a bit, the critical speed $v_{c}$ is that speed at which all blood that enters a region at one RF pulse has left by the next RF pulse. In that case, the $z$-region between 0 and TH is said to be refreshed by fully "unsaturated" spins and there has been complete inflow of new blood. (The adjective "unsaturated" describes spins whose longitudinal magnetization is at the thermal equilibrium value, $M_{0}$.) The result is a higher signal from blood relative to stationary tissue since the refreshed blood has seen fewer RF pulses and is less saturated. (In this context, a "fully saturated" set of spins has the equilibrium longitudinal magnetization $M_{z e}$ described in UNIT B5.1). The mechanism which leads to the increased blood signal is sometimes referred to as "inflow enhancement."

\section{Approach to equilibrium}

Depending on the flip angle $\theta$, it takes some time for stationary spins in a given isochromat to reach the spoiled equilibrium longitudinal magnetization value of equation B5.1.15 from UNIT B5.1:

$$
M_{z e}=\frac{M_{0}\left(1-E_{1}\right)}{1-q}
$$

with:

$$
q \equiv E_{1} \cos \theta
$$

where $E_{1} \equiv e^{-T_{R} / T_{1}}$. For a finite number of RF pulses, the equilibrium value is not yet reached and, just before the $(j+1)$ st pulse (or at the end of the $j$ th repetition period, denoted by $j T_{R}^{-} \equiv j^{-}$), the longitudinal magnetization is instead given by Equation B5.1.22 from UNIT B5.1:

$$
M_{z}\left(j^{-}\right)=M_{z e}+q^{j}\left(M_{0}-M_{z e}\right) \quad j \geq 0
$$

Since $|q|<1$, Equation B7.3.5 yields yields Equation B7.3.3 in the limit, $j \rightarrow \infty$. This describes the signal behavior for both stationary spins and moving spins as observed in their moving frame of reference. That is, independent of whether or not a spin is moving, its longitudinal magnetization is found from Equation B7.3.5 as long as the spin is affected by exactly $j$ cycles, each of flip angle $\theta$. The relevant transverse magnetization just after the $(j+1)$ st RF pulse is found from $M_{z}\left(j^{-}\right) \sin \theta$. Signal curves for various $\theta$ are shown in Figure B7.3.2 and exhibit the expected reduction (saturation) with an increase in the number of RF pulses.

\section{Two-dimensional (2-D) imaging}

We shall carry through a detailed example with $v<v_{c}$ for imaging one slice in a 2-D imaging experiment where blood flow is perpendicular to the slice as depicted in Figure B7.3.3. The experiment consists of a set of $p$ pulses, each a time $T_{R}$ apart. Consider a subslice or "velocity segment" defined with thickness $v T_{R}$, the distance the blood flows in one repeat time.

Except for edge effects, a fixed or moving velocity segment within the overall slice sees each additional RF pulse administered. For convenience, in the analysis to follow, assume that an exact integer $n$ of velocity segments fit into the slice. We have:

Motion Artifacts, Motion Compensation, and Magnetic Resonance Angiography

B7.3.3 


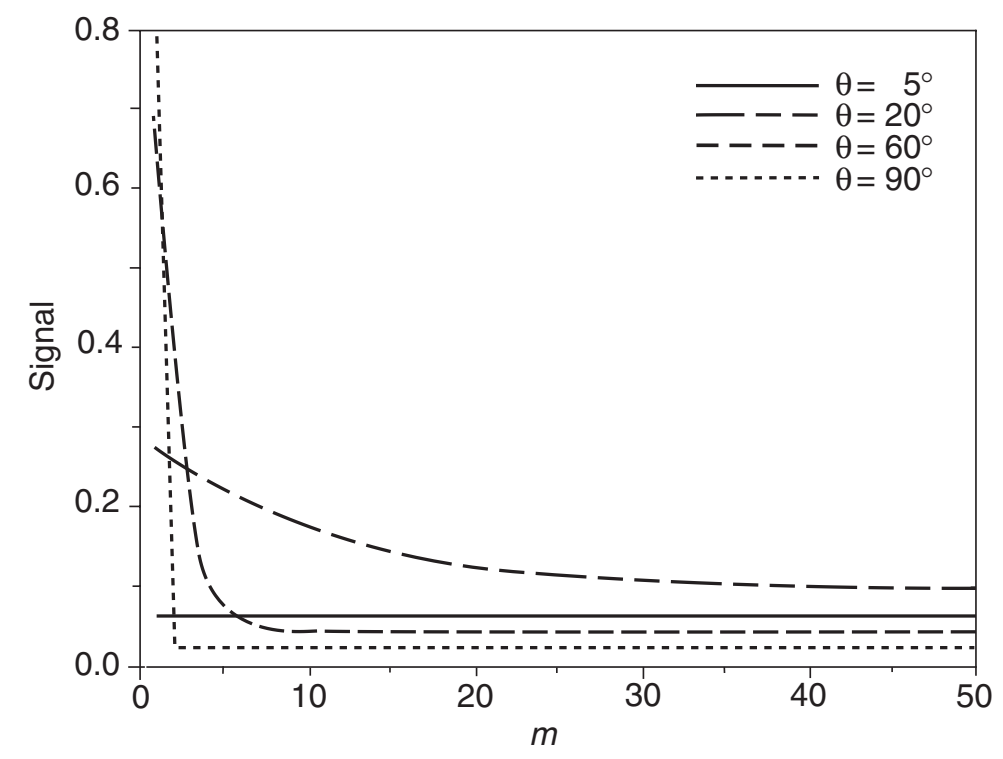

Figure B7.3.2 The signal obtained by multiplying Equation B7.3.5 by $\sin \theta$ is displayed as a function of the number of RF repetition periods (where $\theta$ is the flip angle). The parameter $m$ represents the number of RF pulses and it is equivalent to $j+1$ where $j$ is the parameter in Equation B7.3.5. The value $T_{1}=1200 \mathrm{msec}$ has been chosen as relevant to blood at $1.5 \mathrm{~T}$ along with $T_{R}=$ $40 \mathrm{msec}$ (or for any experiment with a ratio of $T_{R} / T_{1}=1 / 30$ ). The transverse magnetization is assumed to be zero (i.e., spoiled in some way) at the end of each $T_{R}$. Notice the familiar result where the $\pi / 2$ pulses reach equilibrium immediately. When motion is considered, the abscissa is linearly related to the distance $d=j v T_{\mathrm{R}}$ that fresh blood has traveled into a 3-D volume being imaged during the time of the first $j$ RF pulse cycles. Thus, its signal saturates in time, as the moving blood "velocity segment" sees more and more pulses, fading into the background if the background is stationary tissue with the same $T_{1}$. Although the high flip angle examples start off with more magnetization for low $m$, there is a rapid cross-over past which the flip angle giving the highest signal can be associated with the Ernst angle.

$$
n \equiv\left(\mathrm{TH} / v T_{R}\right) \quad \text { with } n \geq 2
$$

(since the $n=1$ case corresponds to the $v=v_{c}$ case). For any integer $n$, all spins in a given fixed velocity segment (and, strictly speaking, provided one of its edges is coincident with a slice edge at some RF pulse instant) will experience the same number of RF pulses as time goes on. This subdivision of the imaged slice makes it possible to follow the signal behavior of the entire slice in the presence of the blood flow. And, in general, for arbitrary, but large values of $\mathrm{TH} / v T_{R}$, a subdivision according to the nearest integer will lead to a reasonable approximation.

Before any RF pulses, the full thermal equilibrium magnetization value $M_{0}$ will be available, and the signal from the slice just after the first RF pulse is given by $M_{0} \sin \theta$. The spins in the slice just before the second RF pulse will have two populations, one of which is still inside occupying a fraction $\left(1-v T_{\mathrm{R}} / \mathrm{TH}\right)$ of the slice, and the other from incoming blood occupying the remaining fraction $v T_{\mathrm{R}} / \mathrm{TH}$ (after one $T_{\mathrm{R}}$, blood from region A has moved into region B of Fig. B7.3.3). Hence, the signal from the same slice just after the action of the second pulse is given by the sum of these two contributions.

Magnetic Resonance Angiography

More generally, the velocity segment of blood that occupied region A just before the first RF pulse, will have moved, just before the $p$ th pulse, an additional distance $(p-1) v T_{R}$.

B7.3.4 
A

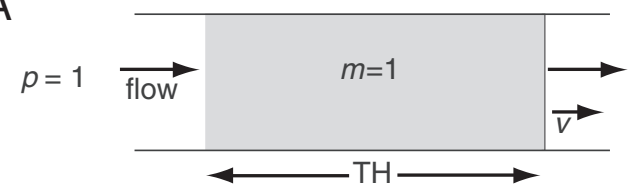

B

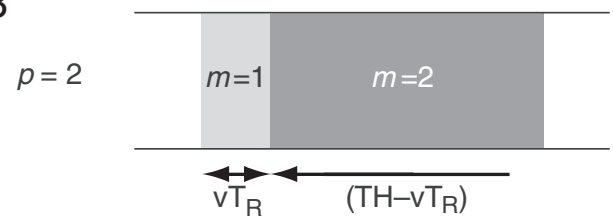

C

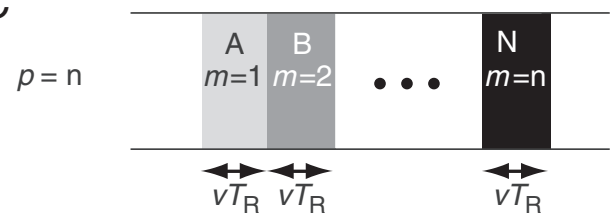

D

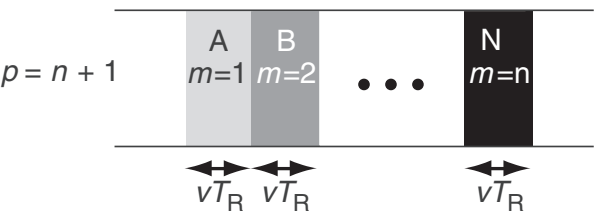

$E$

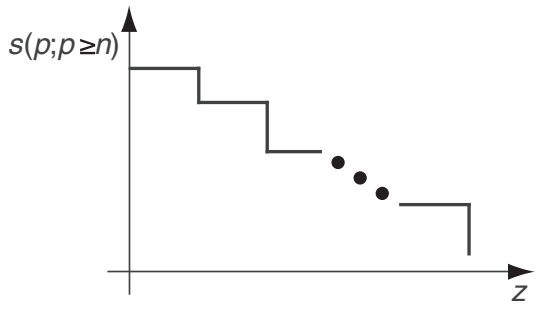

Figure B7.3.3 Saturation of spins flowing into a slice is demonstrated in this figure as a function of the number of RF pulses $p$. (A) At the instant after the first RF pulse, all excited spins have equal transverse magnetization. The index $m \equiv j+1$ (used in Equation B7.3.5) indicates the number of RF pulses seen in a given velocity segment. (B) At the instant after the second pulse, a fraction of excited blood has moved a distance $v T_{R}$ and the new blood has replaced that space. The old (new) blood has experienced 2 RF pulses (1 RF pulse). A steady state is obtained where spins with longitudinal magnetization $M_{0}$ freshly enter the slice between RF pulses into the first of the $n$ fixed velocity segments along the direction of flow as illustrated in parts (C) and (D) for the $n$th and $(n+$ 1)st pulse. The regions with the lower $m$ labels have the higher signal (lighter shading), thanks to the inflow of fresh blood, while the darker shading represents lower (more saturated) signal. The signal behavior as a function of position of the artificially created velocity segments in the direction of flow in the steady state is shown schematically in part (E).

At the instant after the $p$ th pulse, it thus occupies the $p$ th fixed velocity segment of the slice and has experienced $p$ pulses. And at the instant that the $n$th pulse has occurred, this particular spin population will have experienced a total of $n$ pulses, and occupy the fixed velocity segment labeled $N$. At the other end, the spin population that moved just prior to section A before the $(n-1)$ st pulse to occupy section A will have experienced only one RF pulse just after the $n$th RF pulse of the experiment. Altogether, there will be $n$ spin population fixed velocity segments (each of thickness $v T_{\mathrm{R}}$ ) experiencing $m=1,2, \cdots n$ RF pulses, respectively, the number $m$ increasing with position $z$ in the direction of the flow. The $m$ th velocity segment sees $m$ pulses when $n$ RF pulses have been administered

Motion Artifacts,

Motion

Compensation,

and Magnetic

Resonance

Angiography

B7.3.5

Supplement 7 

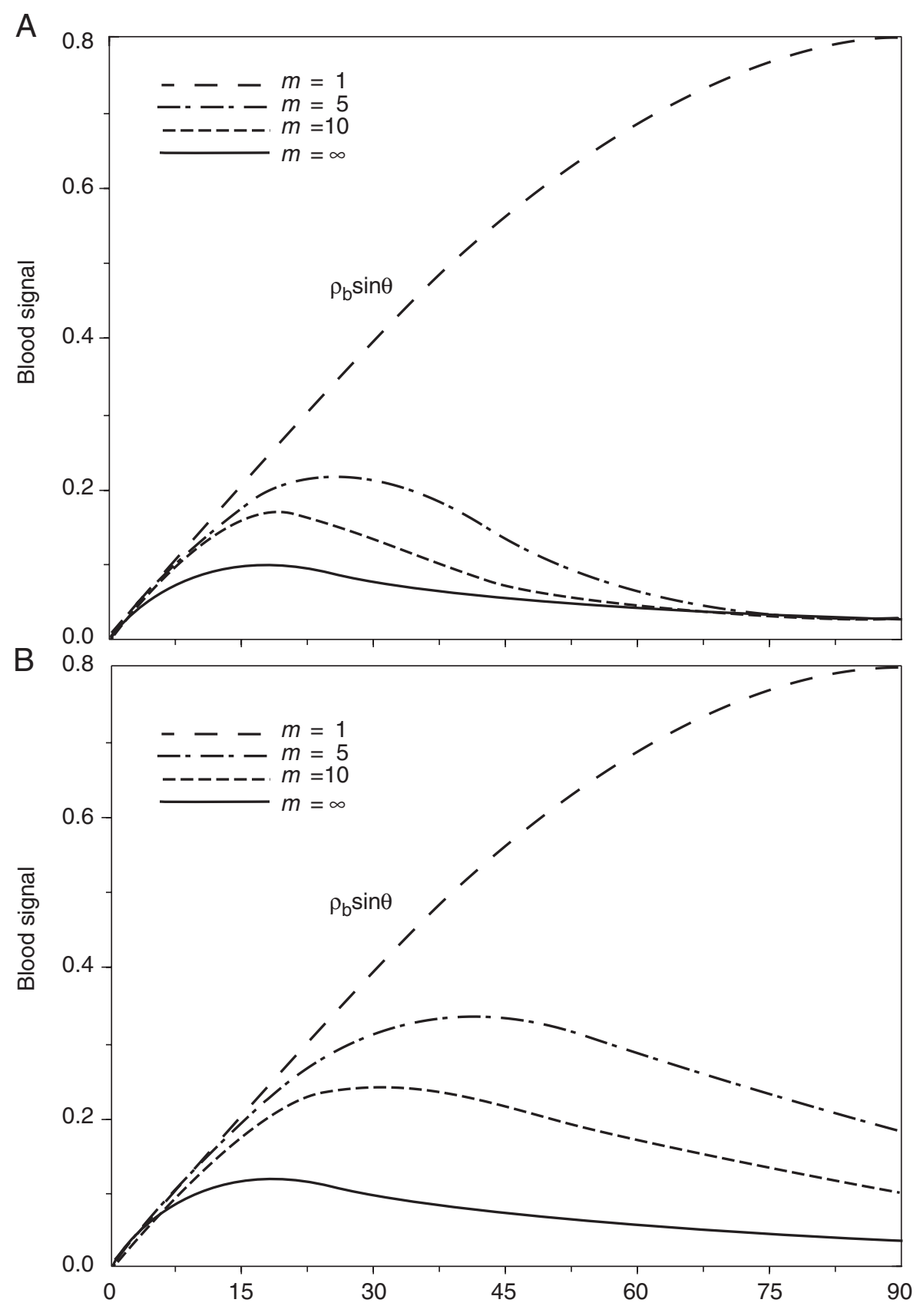

Flip angle (degrees)

Figure B7.3.4 Signal from blood as a function of flip angle $\theta$ with $T_{\mathrm{R}}=40 \mathrm{msec}$ and $T_{1}=1200$ msec for (A) a thin slice (for blood in 2-D or 3-D or any stationary tissue segment) and (B) the full 2-D slice, showing the dependence on the number of pulses. In (A), the number of pulses seen by the thin slice is $m$ and, in (B), the number of pulses administered to the full slice is greater than or equal to $n$. The slices are perpendicular to the flow. The normalization is such that, for one thin slice and $m=1$, the signal is $\rho_{b} \sin \theta$ where $\rho_{b}$ is stylized as 0.8 relative to the spin density of water. 
A

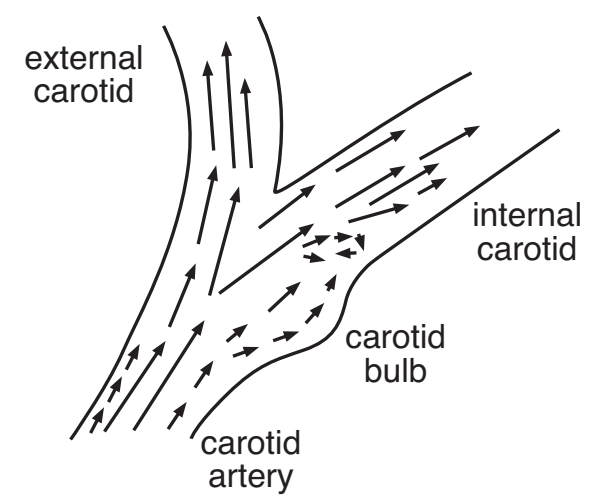

B

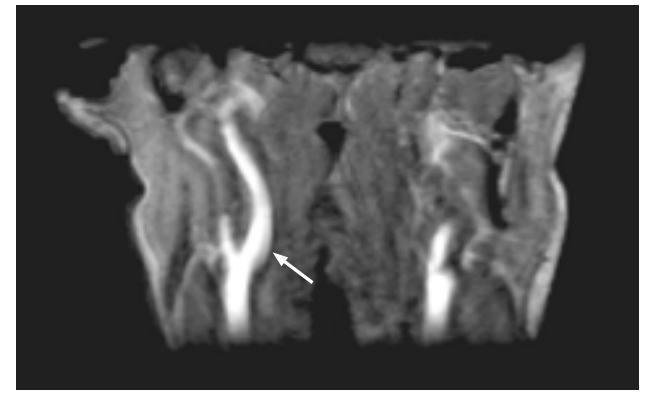

Figure B7.3.5 (A) Flow pattern seen at some point in a vessel such as that for the carotid artery branching into the internal and external carotids. The carotid bulb area can have vortex flow which is a circulating flow pattern where spins remain longer than those spins flowing straight through the vessel. (B) A coronal slice through the carotid artery of a volunteer showing the "streamlining" effect where fast flow is bright and slow flow (as in the carotid bulb) is darker. Imaging parameters: (B) $T_{R} / T_{E}=20.1 \mathrm{msec} / 4.8 \mathrm{msec}, T_{s}=10.24 \mathrm{msec}, \Delta x \times$ $\Delta y \times \Delta z=1.0 \mathrm{~mm} \times 1.0 \mathrm{~mm} \times 1.0 \mathrm{~mm}, N_{x} \times N_{y} \times N_{z}=256 \times 256 \times 100, \theta=20^{\circ}, N_{\text {acq }}=1, \tau_{R F}=1.28 \mathrm{msec}, G_{s S}$ $=2.2 \mathrm{mT} / \mathrm{m}$.

to the full slice. Notice that the longitudinal magnetization just before the $p$ th pulse in each segment determines its contribution to the signal just after the $p$ th pulse. Thus the relevant values of the segment magnetization depend on $m-1$ pulses as seen in the individual terms in the sum in Equation B7.3.5. By the time the number of pulses reaches the number $n$ of fixed velocity segments, a steady signal is obtained from the blood vessel and is given by a sum over all the velocity segments and their respective contributions derived from Equation B7.3.5.

A comparison of the saturation signal of a given velocity segment with that summed over whole slice is shown in Figure B7.3.4A and Figure B7.3.4B, respectively. For the saturated or steady behavior of the whole slice, recall that the number $p$ of pulses must be greater than or equal to $n$, a parameter determined by the velocity of the spins and the slice thickness.

\section{Three-dimensional (3-D) imaging}

In 3-D imaging, we may be able to obtain spatial information on the scale of the individual velocity segments of a given slab. Recall an advantage of 3D imaging is the ability to obtain thin slices for high resolution imaging and short $T_{\mathrm{E}}$ whereas a thin slice 2-D experiment requires a long RF pulse time to create a sharp slice profile and the thickness is limited by the available gradient strength. However, the saturation encountered as the blood propagates into the slab remains an important issue in 3-D image contrast.

The analysis of the signal for a velocity segment of the slab is the same as for the 2-D slice. Equation B7.3.3 remains appropriate for the signal from a given segment in a 3-D slice where $m$ represents both the velocity segment number and the number of RF pulses experienced by that segment. (In practically, it is quite common to apply a series of $n$ pre-pulses, or dummy cycles, prior to collecting the data so that the blood in each partition has reached the equilibrium condition shown in Fig. B7.3.3C.) The segmentation in Figure B7.3.3 and the signal plots in Figure B7.3.2 and Figure B7.3.4A apply here as well, and, as the blood moves into the slab, it becomes increasingly saturated. While at large flip angles (close to $90^{\circ}$ ) only a few pulses are needed for saturation and the signal of the

Motion Artifacts, Motion Compensation, and Magnetic Resonance Angiography

\section{B7.3.7}

Supplement 7 
A

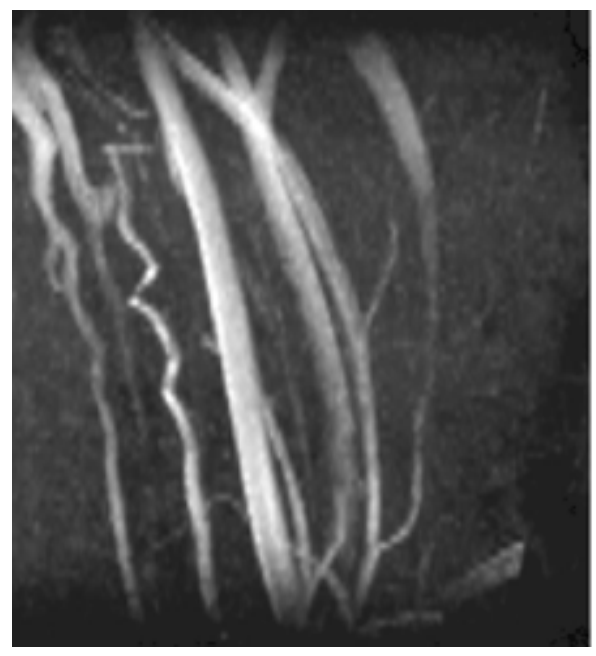

C

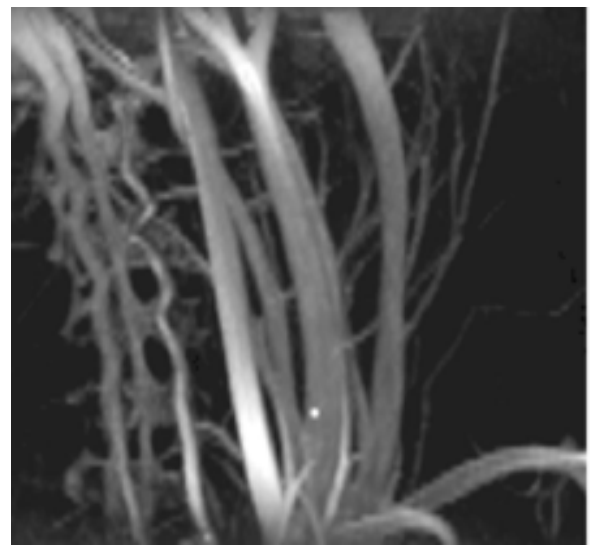

B

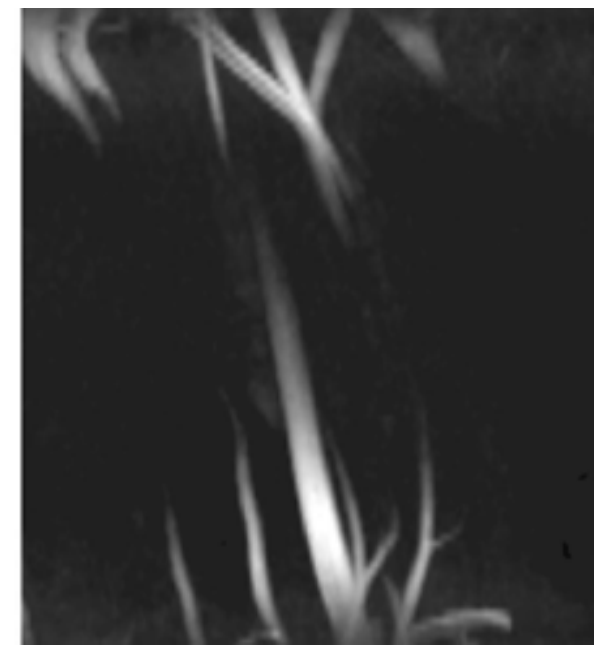

D

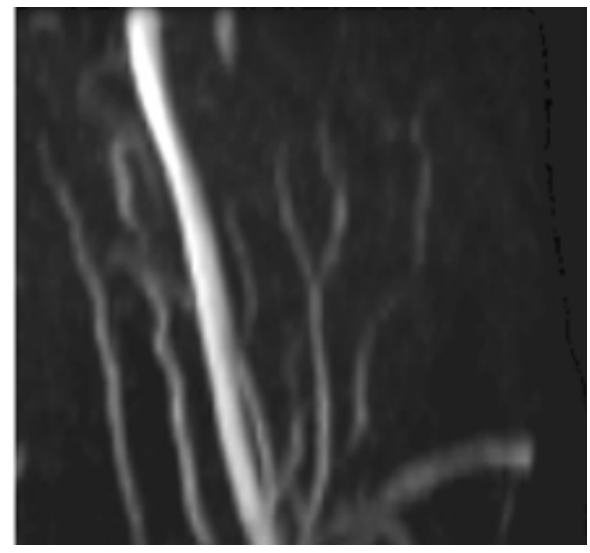

Figure B7.3.6 A short $T_{\mathrm{R}}$ 3-D transverse data acquisition of blood flowing through the neck of a pig. The images shown are constructed by a coronal maximum intensity projection of a stack of the transverse slices. (A) The signal intensity is fairly constant in a low flip angle $\left(15^{\circ}\right)$ acquisition. (B) A powerful demonstration of the saturation effect is obtained when the same region is imaged with a large flip angle such as $55^{\circ}$. Note the decrease in signal of the arteries from bottom upwards and of the veins from top downwards due to spin saturation. Smaller vessels with slower flow are so saturated that they are not even visible on the MIP. (C) A "post-contrast" MIP of the vessels and (D) a "pre-contrast" MIP from a 2-D MRA acquisition are shown for comparison (see the discussion of the use of contrast agents to obtain an enhanced vascular signal). Imaging parameters: (A) $T_{\mathrm{R}} / T_{\mathrm{E}}$ $=21.0 \mathrm{msec} / 6.0 \mathrm{msec}, T_{s}=12.8 \mathrm{msec}, \Delta x \times \Delta y \times \Delta z=0.39 \mathrm{~mm} \times 0.78 \mathrm{~mm} \times 1.0 \mathrm{~mm}, N_{x} \times N_{y} \times$ $N_{z}=512 \times 192 \times 96, \theta=15^{\circ}, N_{\text {acq }}=1, \tau_{R F}=1.024 \mathrm{msec}, G_{s s}=1.72 \mathrm{mT} / \mathrm{m}$; (B), (C) $\theta=55^{\circ}$; (D) $T_{\mathrm{R}} / T_{\mathrm{E}}=25.0 \mathrm{msec} / 9.0 \mathrm{msec}, T_{s}=5.12 \mathrm{msec}, \Delta x \times \Delta y \times \Delta z=0.78 \mathrm{~mm} \times 1.25 \mathrm{~mm} \times 3.0 \mathrm{~mm}, N_{x} \times$ $N_{y}=256 \times 160, \theta=35^{\circ}, N_{\text {acq }}=1, \tau_{R F}=2.048 \mathrm{msec}, G_{s s}=8 \mathrm{mT} / \mathrm{m}$. 
A

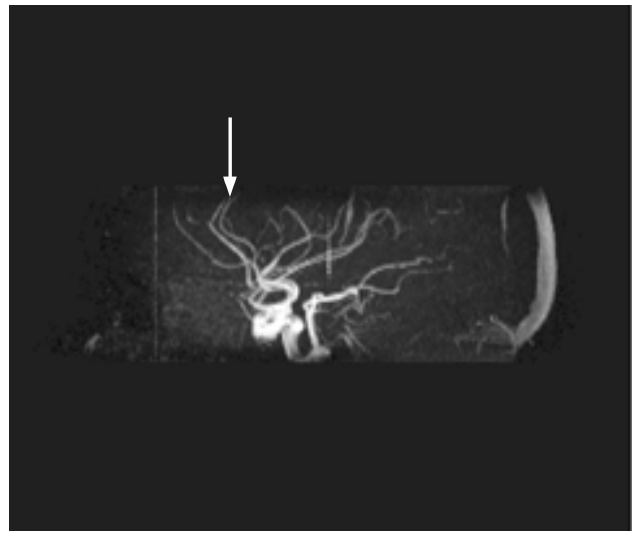

B

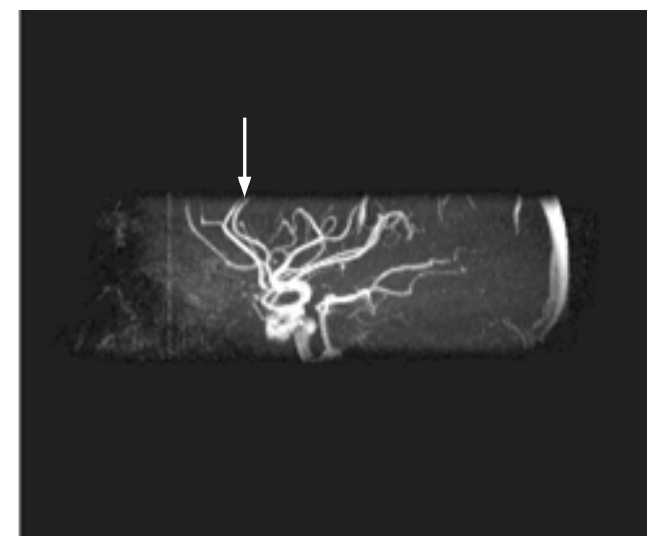

Figure B7.3.7 A spatially varying RF pulse acquisition is used to overcome the saturation effect as a function of distance along the slice select direction. Here, flow is assumed to be occurring predominantly in the slice select direction (in the up/down direction here). (A) A thick-slice 3-D MRA acquisition shows the saturation effect of the arteries toward the top of the head (arrows) whereas (B), the spatially varying RF pulse acquisition of the same slice, shows little visible saturation effect. The arrows in $(A)$ and $(B)$ point to a vessel exiting the top of the slice which is clearly visible in (B), but less so in (A). Imaging parameters: $(A)$ $T_{\mathrm{R}} / T_{\mathrm{E}}=25.0 \mathrm{msec} / 6.75 \mathrm{msec}, T_{s}=10.24 \mathrm{msec}, \Delta x \times \Delta y \times \Delta z=0.5 \mathrm{~mm} \times 1.0 \mathrm{~mm} \times 1.0 \mathrm{~mm}, N_{x} \times N_{y} \times N_{z}$ $=512 \times 192 \times 70, \theta=15^{\circ}, N_{\text {acq }}=1, \tau_{R F}=1.024 \mathrm{msec}, G_{S S}=3.14 \mathrm{mT} / \mathrm{m} ;(B) \theta=15^{\circ} ;$ RF pulse with linearly increasing flip angle as a function of $z$ reaching $35^{\circ}$ at the top edge of the excited slab, $N_{\text {acq }}=1$.

blood is small; at flip angles near the Ernst angle (i.e., $\cos ^{-1} E_{1}$ ), the signal does not reach the steady-state level even if the spins have experienced as many as $10 \mathrm{RF}$ pulses. When the flip angle is equal to the Ernst angle, the signal is maximum. When the flip angles are small, the longitudinal magnetizations are only slightly tipped for small $\theta$ so that they do not need time to recover. In this case, even the image of the slow flowing blood can be brighter than the image of the same blood when a $90^{\circ}$ pulse is used.

Consider another perspective for the result that smooth, fast blood flow will tend to produce a larger signal than will slower blood flow or a stationary background (brain parenchyma or muscle, for example). Figure B7.3.5 exhibits a realistic spatial velocity distribution for the carotid artery and its bifurcation. The length of each vector represents how far the blood has traveled between each RF pulse and the direction represents the direction of the flow of a spin at that position. For laminar flow, the center or faster flow will see the fewest number of RF pulses and will be brightest at any cross-section (see Fig. B7.3.5B).

An example of 3-D imaging of blood flowing up through the neck of a pig is shown in Figure B7.3.6. The signal remains constant along the blood vessels for low flip angles (faster recovery) in Figure B7.3.6A, where the background is suppressed by "magnetization transfer contrast" (or "magnetization transfer saturation") below. More saturation is evident in Figure B7.3.6B for a larger flip angle, and the TOF effect is clearly present. The suppression of smaller vessels, in which the flow is slower, is also observed in this figure.

Motion Artifacts,

Motion

Compensation, and Magnetic

Resonance

Angiography

\section{B7.3.9}

Supplement 7 
A

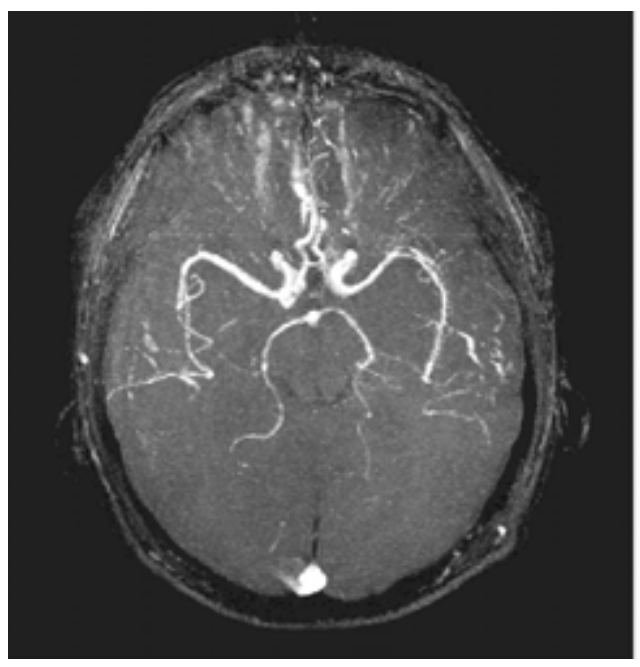

C

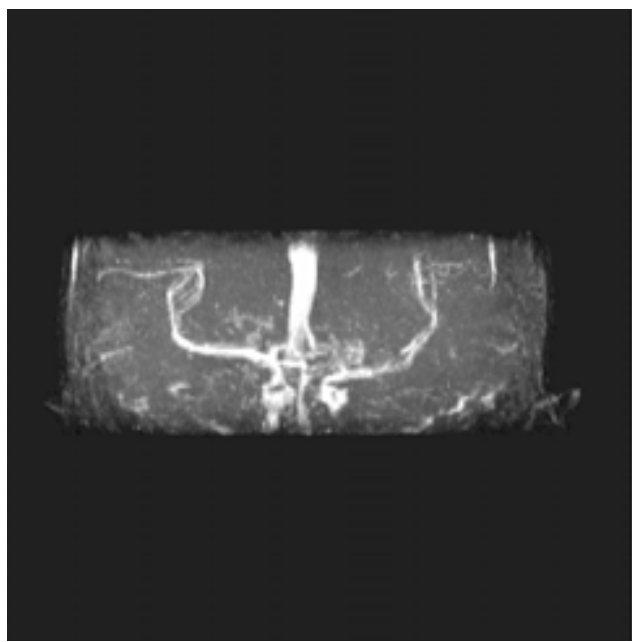

$\mathrm{B}$

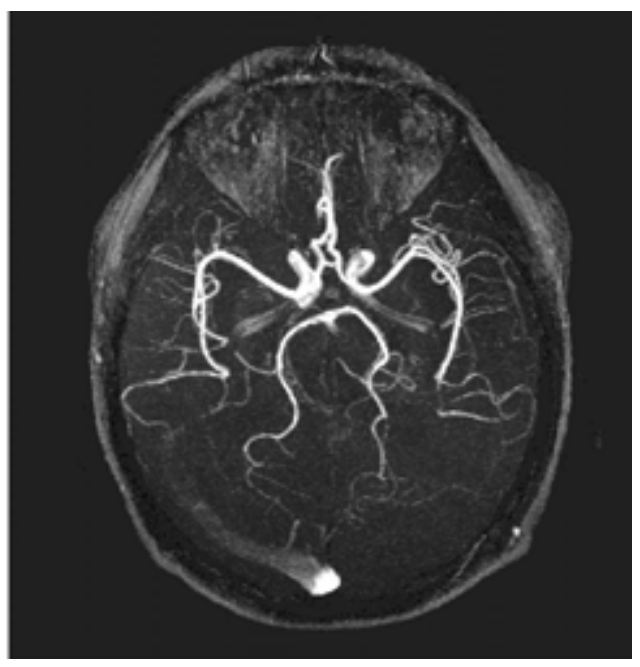

$\mathrm{D}$

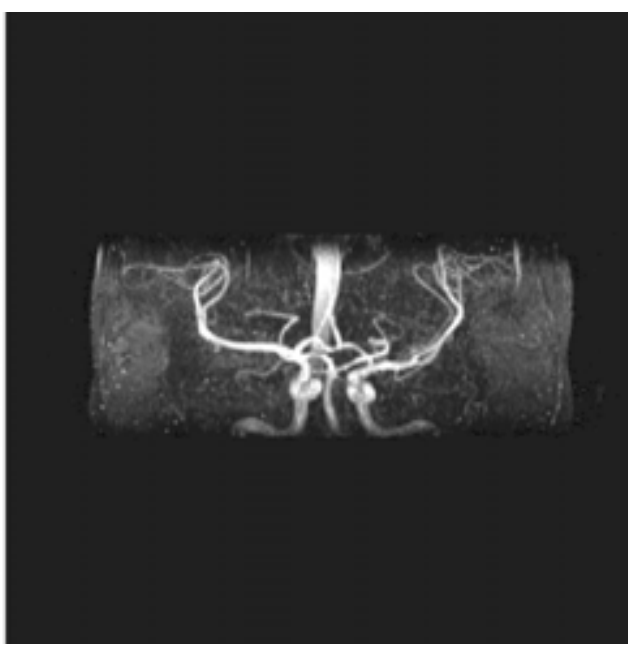

Figure B7.3.8 "Magnetization transfer saturation" can be utilized to suppress the background brain tissues preferentially to highlight even the more readily saturated small vessels. In this case, a spin density-weighted acquisition can be used to obtain enhanced MR angiograms. (A) Vessels are more easily viewed in a 3-D MRA acquisition by performing an MIP. This particular example was obtained using a $T_{1}$-weighted sequence. This highlights the large vessels, which have faster flow, while the small vessels are invisible. (B) An MIP from an MRA acquisition using an MTS pulse prior to each excitation pulse now highlights the small vessels as well. Note the suppression of most GM-WM contrast in the background. A coronal view in (C) and (D) also reveals improved vessel visibility with the use of MTS. Imaging parameters: (A), (C) $T_{R} / T_{E}=34.0$ $\mathrm{msec} / 6.5 \mathrm{msec}, T_{s}=12.8 \mathrm{msec}, \Delta x \times \Delta y \times \Delta z=0.5 \mathrm{~mm} \times 1.0 \mathrm{~mm} \times 1.0 \mathrm{~mm}, N_{x} \times N_{y} \times N_{z}=512 \times 192 \times 70$, $\theta=15^{\circ}$, with no MTS preparation pre-pulse, $N_{\text {acq }}=1, \tau_{R F}=1.536 \mathrm{msec}, G_{s s}=2.07 \mathrm{mT} / \mathrm{m} ;(B),(D) \theta=15^{\circ}$, with MTS preparation pre-pulse. 
A

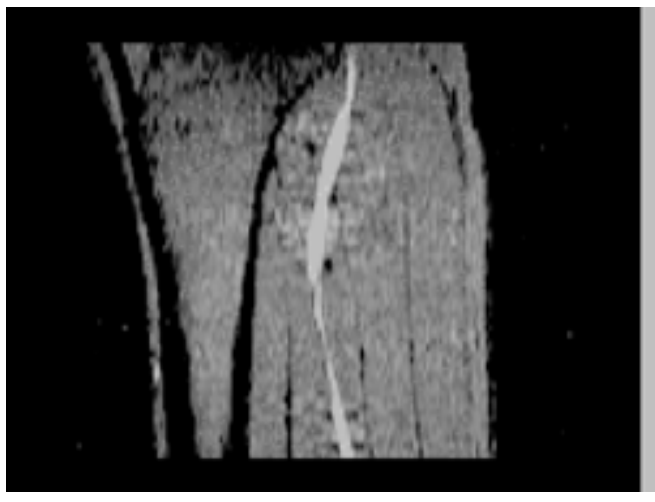

B

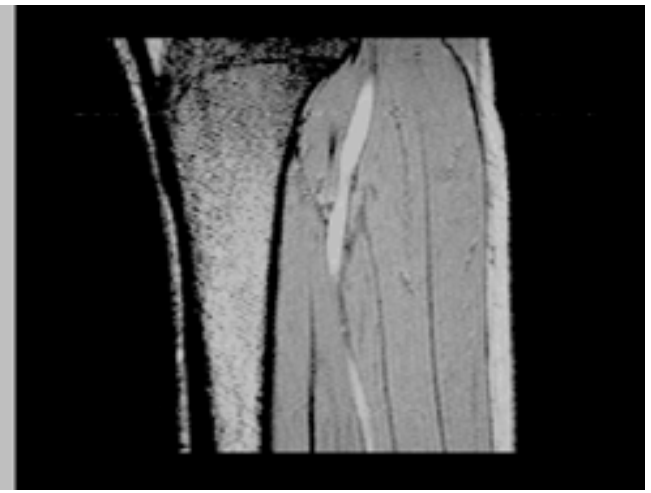

Figure B7.3.9 An MRA acquisition in the leg obtained by using a long $T_{\mathrm{E}}$ acquisition utilizing the longer $\mathrm{T}_{2}$ of blood in comparison with other surrounding tissues. (A) A conventional 2-D MRA sequence which highlights the slow flow, thanks to the TOF effect. (B) A similar suppression of the surrounding tissues in comparison with the blood is obtained by using a long $T_{\mathrm{E}}$ acquisition. Although there is still some time-of-flight effect present, venous blood has a shorter $T_{2}{ }^{*}$ than arterial blood and is further suppressed this way. No inflow is required to obtain bright arterial signal relative to muscle when a longer $T_{E}$ is used. The local MIP shown in part (B) was obtained from images that were collected using a $T_{E}$ of $24 \mathrm{msec}$, a $T_{\mathrm{R}}$ of $46 \mathrm{msec}$ and a flip angle of $25^{\circ}$. Imaging parameters: (A) $T_{\mathrm{R}} / T_{\mathrm{E}}=25 \mathrm{msec} / 9 \mathrm{msec}, T_{s}=5.12 \mathrm{msec}, \Delta x \times \Delta y \times \Delta z=1.0$ $\mathrm{mm} \times 1.0 \mathrm{~mm} \times 2.0 \mathrm{~mm}, N_{x} \times N_{y}=256 \times 160, \theta=35^{\circ}, N_{\text {acq }}=1, \tau_{R F}=2.048 \mathrm{msec}, G_{s s}=12.0 \mathrm{mT} / \mathrm{m} ;(\mathrm{B})$ $T_{\mathrm{R}} / T_{\mathrm{E}}=46 \mathrm{msec} / 24 \mathrm{msec}, T_{s}=10.24 \mathrm{msec}, \Delta x \times \Delta y \times \Delta z=0.5 \mathrm{~mm} \times 1.0 \mathrm{~mm} \times 1.0 \mathrm{~mm}, N_{x} \times N_{y} \times N_{z}=512$ $\times 160 \times 132, \theta=25^{\circ}, N_{\text {acq }}=1, \tau_{R F}=2.56 \mathrm{msec}, G_{s s}=0.31 \mathrm{mT} / \mathrm{m}$.

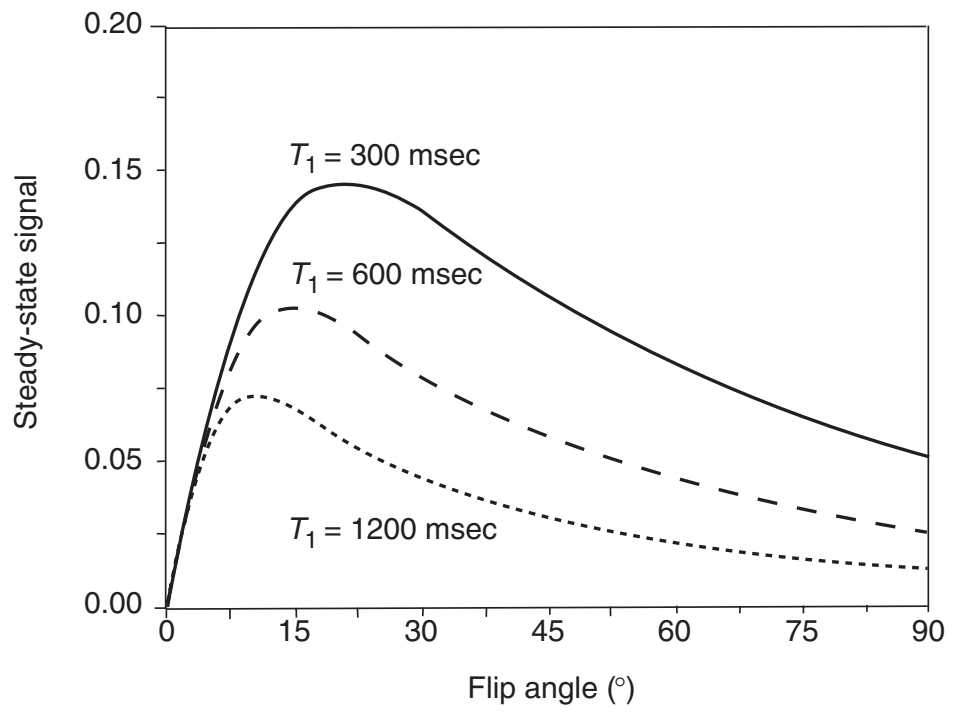

Figure B7.3.10 Plot of the blood signal for $m=\infty$, and $T_{\mathrm{R}}=20 \mathrm{msec}$ with $T_{1}=1200 \mathrm{msec}, 600$ msec, and $300 \mathrm{msec}$. Even blood which sees a large number of RF pulses with $T_{1}=300 \mathrm{msec}$ still has a signal much higher than that for tissue with $T_{1}=1200 \mathrm{msec}$. For this reason, $T_{1}$-reducing contrast agents play a key role in enhancing vascular information.

Motion Artifacts, Motion Compensation, and Magnetic Resonance Angiography

\section{B7.3.11}




\section{TOF Contrast, Contrast Agents, and Spin Density $/ T_{2}{ }^{*}$ Weighting}

Now that we understand the behavior of the blood signal as it penetrates deeper into an imaging slice, the next goal is to further increase its signal relative to the background. This can be done by a variety of means. First, the signal can be preferentially enhanced deeper into the slab to offset the TOF saturation as a function of position. This is accomplished by varying the flip angle as a function of position along the slice select direction, typically by linearly increasing the flip angle along the direction of flow (see Fig. B7.3.7). Second, the signal from the background tissue can be suppressed by using "magnetization transfer saturation." (Magnetization transfer saturation, MTS, is a means by which spins residing outside the normal on-resonant frequency are slowly saturated. These spins then exchange into the on-resonant range but now have little signal left. This suppression is best-achieved with specially designed RF pulses whose duration is long enough to cause a significant effect.) The MTS pulse suppresses white matter (WM) and gray matter (GM) leading to enhanced contrast from the blood which is essentially not suppressed (see Fig. B7.3.8). Third, contrast between blood and background tissue can be increased by using a long- $T_{\mathrm{E}}$ scan (see Fig. B7.3.9). Fourth, a $T_{1}$-reducing contrast agent can be used to increase the signal from blood, the next subject to be discussed.

\section{Contrast agents}

The use of $T_{1}$-reducing contrast agents that can be injected into the blood stream offers the opportunity to enhance signal selectively from blood without altering signal from the surrounding tissue. Reducing $T_{1}$ will lead to an increase in signal-to-noise ratio (SNR) for both magnitude and phase imaging. In most imaging applications, the contrast agent is assumed to be intravascular (i.e., it stays within the blood vessel). To appreciate the significance of using a contrast agent for MRA, the signal is plotted as a function of flip angle at steady state for several values of $T_{1}$ corresponding to different contrast agent doses (see Fig. B7.3.10). The value of $T_{1}$ after the injection of the contrast agent is $1 / T_{1, \text { post }}$ $=1 / T_{1, \mathrm{pre}}+\alpha[c]$ where $\alpha$ is the relaxivity in units of $\mathrm{mM}^{-1} \mathrm{sec}^{-1}$ and $[c]$ is the concentration of contrast agent in $\mathrm{mM}$. For example, if the $1 / T_{1}$ relaxivity of the agent is $5 \mathrm{mM}^{-1} \mathrm{sec}^{-1}$ and the concentration in the blood is $0.167 \mathrm{mM}$ in the equilibrium state, then the
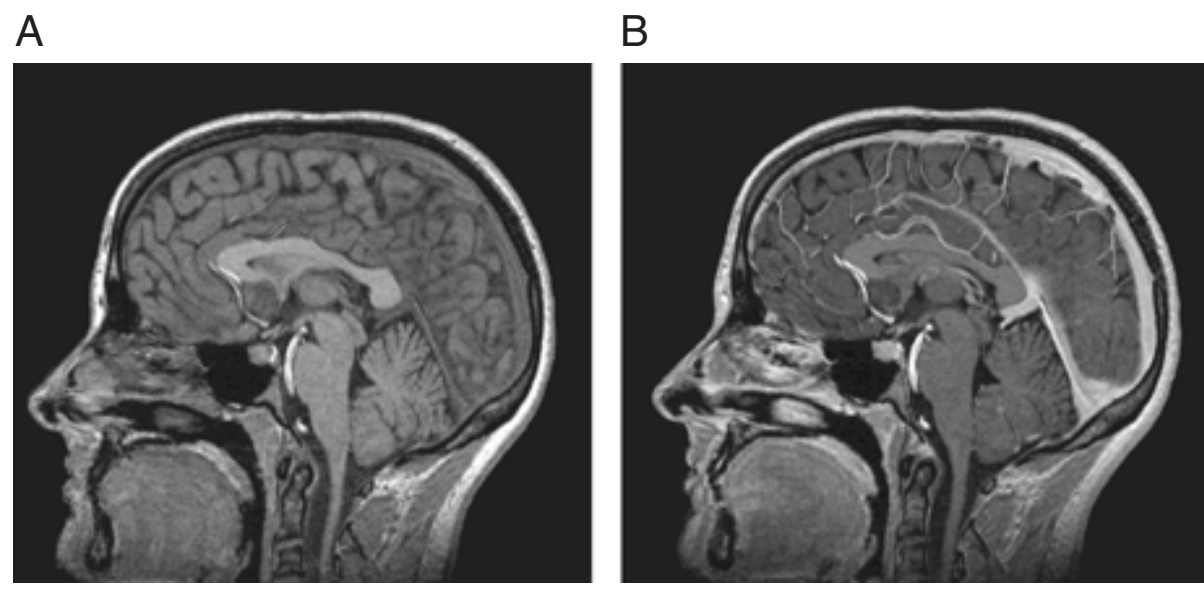

Figure B7.3.11 Images of the brain pre- and post-contrast injection from a 3-D $T_{1}$-weighted data set acquired in a sagittal fashion so that there is essentially no inflow effect except in the large vessels. (A) A single 1-mm-thick sagittal image showing the saturation effect in the pre-contrast image, and (B) enhancement of the blood vessels in the post-contrast image. Imaging parameters: (A), (B) $T_{R} / T_{E}=20.1 \mathrm{msec} / 4.8 \mathrm{msec}, T_{s}=10.24 \mathrm{msec}, \Delta x \times \Delta y \times \Delta z=0.5 \mathrm{~mm} \times 0.5 \mathrm{~mm} \times 1.0 \mathrm{~mm}$, $N_{x} \times N_{y} \times N_{z}=512 \times 512 \times 152, \theta=25^{\circ}, N_{\text {acq }}=1, \tau_{R F}=1.28 \mathrm{msec}, G_{s S}=1.45 \mathrm{mT} / \mathrm{m}$. 
A

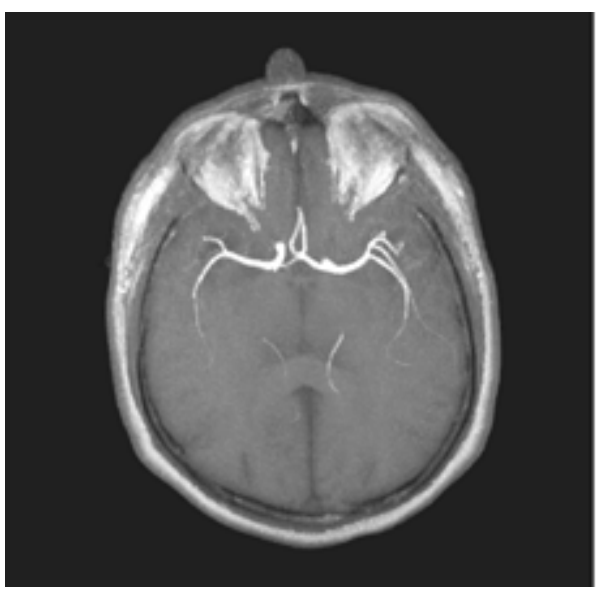

C

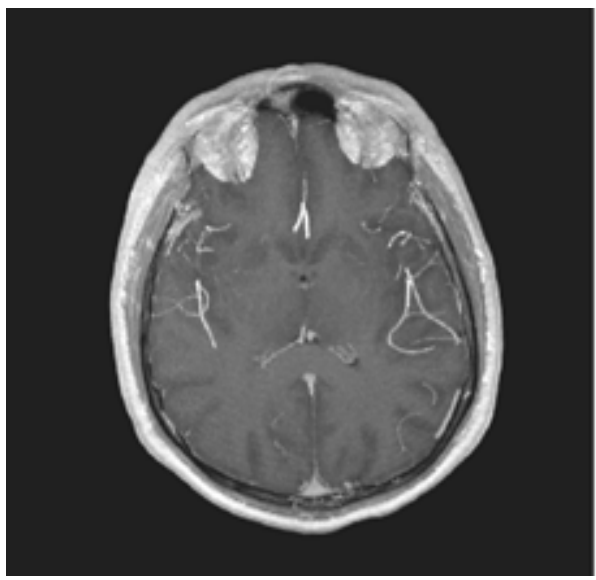

B

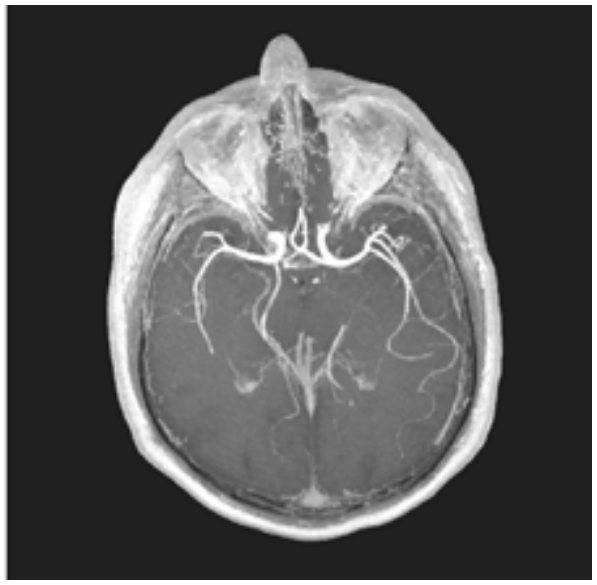

$\mathrm{D}$

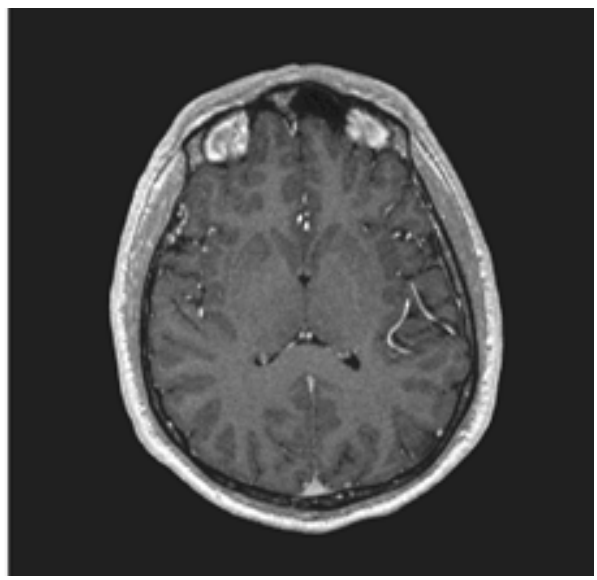

Figure B7.3.12 (A) An MIP image from a pre-contrast 3-D data set. In comparison, (B) the post-contrast 3-D data set MIP over the entire volume shows an abundance of vascular information. However, projection over a smaller volume as in (C) can also be used to study specific parts of the vascular tree. (D) Examining only a single slice better depicts smaller vessels than in the global MIP image shown in part (B). In fact, the vessels look larger in the unprocessed single slices than after the MIP. Imaging parameters: (A), (B) $T_{\mathrm{R}} / T_{\mathrm{E}}=20.1 \mathrm{msec} / 4.8 \mathrm{msec}, T_{s}=10.24 \mathrm{msec}, \Delta x \times \Delta y \times \Delta z=0.5$ $\mathrm{mm} \times 0.5 \mathrm{~mm} \times 24.0 \mathrm{~mm}, N_{x} \times N_{y}=512 \times 384, \theta=25^{\circ}, N_{\mathrm{acq}}=1, \tau_{R F}=1.28 \mathrm{msec}, G_{s s}=1.72 \mathrm{mT} / \mathrm{m}$; (C) $\Delta x \times \Delta y \times \Delta z=0.5 \mathrm{~mm} \times 0.5 \mathrm{~mm} \times 8.0 \mathrm{~mm}$; (D) $\Delta x \times \Delta y \times \Delta z=0.5 \mathrm{~mm} \times 0.5 \mathrm{~mm} \times 1.0 \mathrm{~mm}$.

post-contrast $T_{1}$ of blood would be reduced from $1200 \mathrm{msec}$ to $600 \mathrm{msec}$. With increasing dosage, the $T_{1}$ is reduced more and a higher blood signal results for a given flip angle. This reduction in $T_{1}$ and the concomitant increase in blood signal are well illustrated in Figure B7.3.6C and D, Figure B7.3.11, and Figure B7.3.12, where a large number of blood vessels are enhanced post-contrast injection, and are displayed as bright structures. Even though blood signal is significantly enhanced, and more vessels are apparent on the maximum intensity projection (MIP) in Figure B7.3.12B than Figure B7.3.12A, the MIP operation is still not ideal in that small vessels and low amplitude regions can be significantly suppressed or lost. (The MIP is to take the maximum value along a line of sight of a 3-D data set and write this value to a point in a 2-D display. The result yields a projection image.)

Motion Artifacts, Motion Compensation, and Magnetic Resonance Angiography

\section{B7.3.13}

Supplement 7 


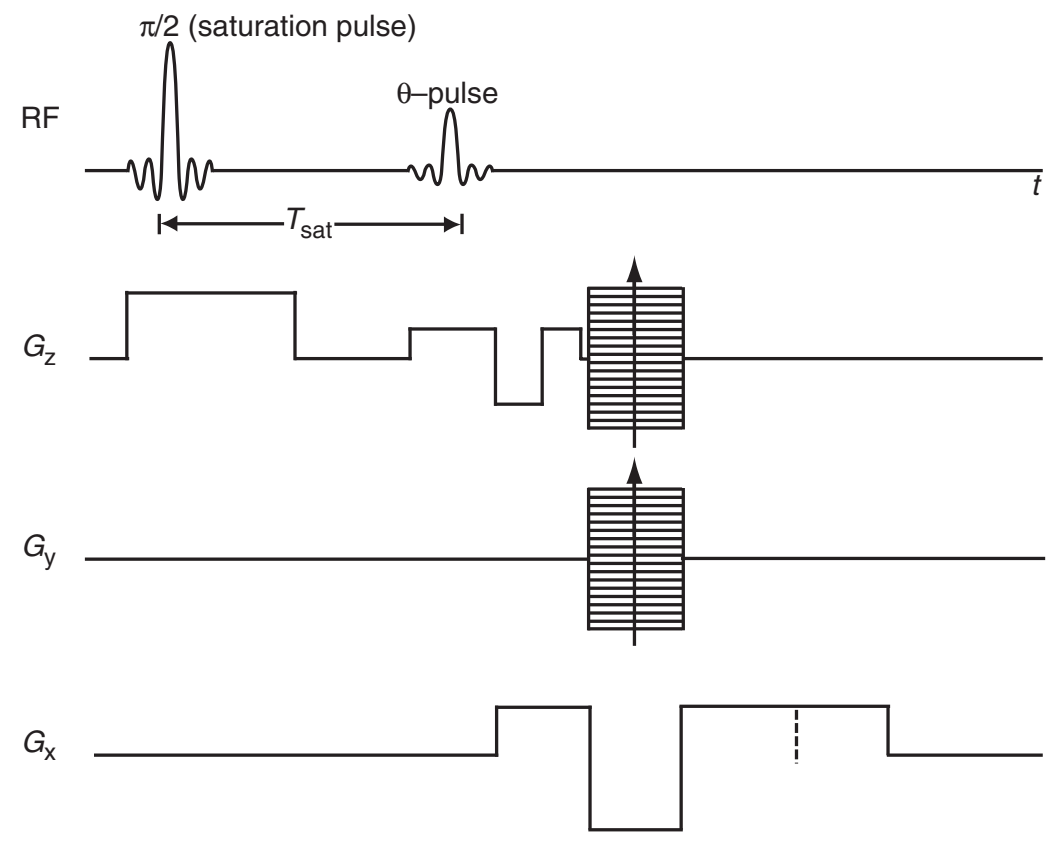

Figure B7.3.13 A typical TOF MRA sequence. The slice select gradient applied during the saturation pulse is kept on afterward to spoil the transverse magnetization created by the saturation pulse. The time between the saturation pulse and the $\theta$-pulse, $T_{\text {sat }}$, is usually on the order of $5 \mathrm{msec}$ to $10 \mathrm{msec}$. The saturation pulse thickness can be varied from a few $\mathrm{mm}$ to many $\mathrm{cm}$ (which is usually the case).

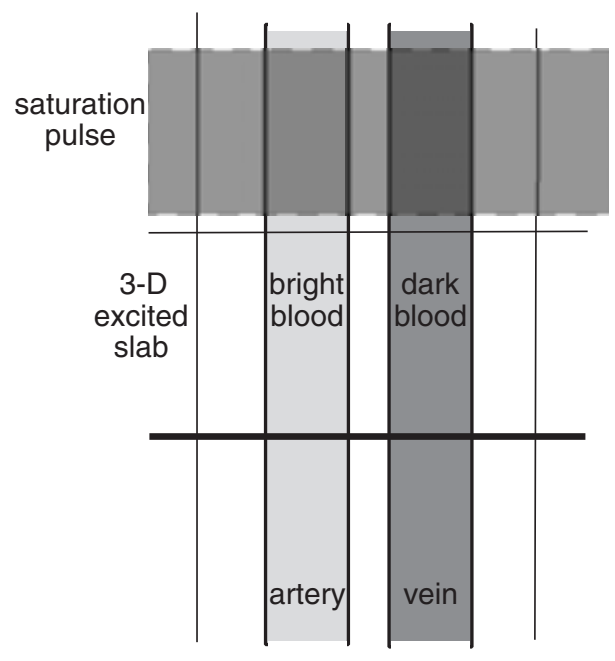

Figure B7.3.14 A schematic of the carotid artery and jugular vein. The first pulse used in the sequence shown in Figure B7.3.13, often set to $90^{\circ}$, is used to saturate the signal from the veins. The second pulse excites the slab of interest. The resulting images in Figures B7.3.15, B7.3.16, and B7.3.17 show dramatically reduced signal from the veins (see those three images for an in vivo demonstration of these concepts). 
A

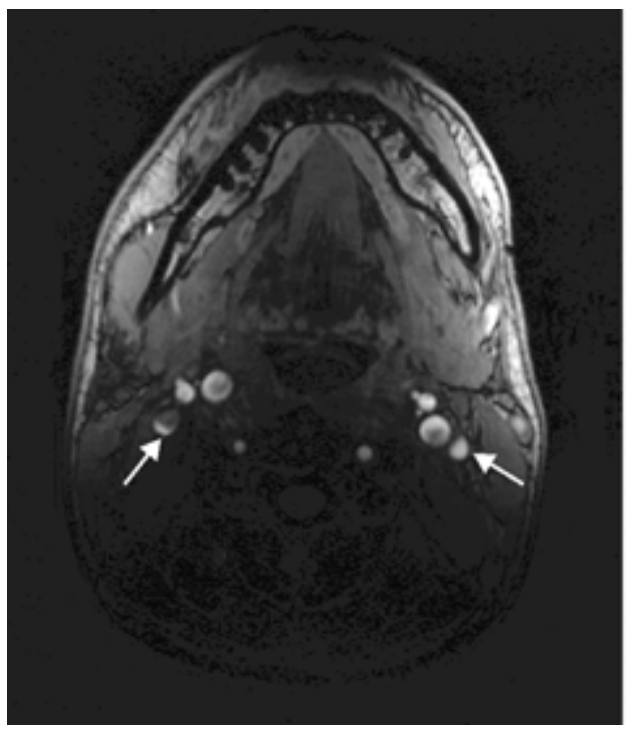

C

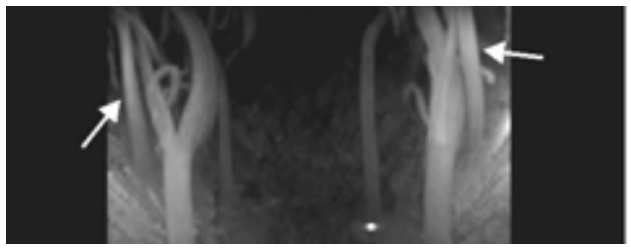

B

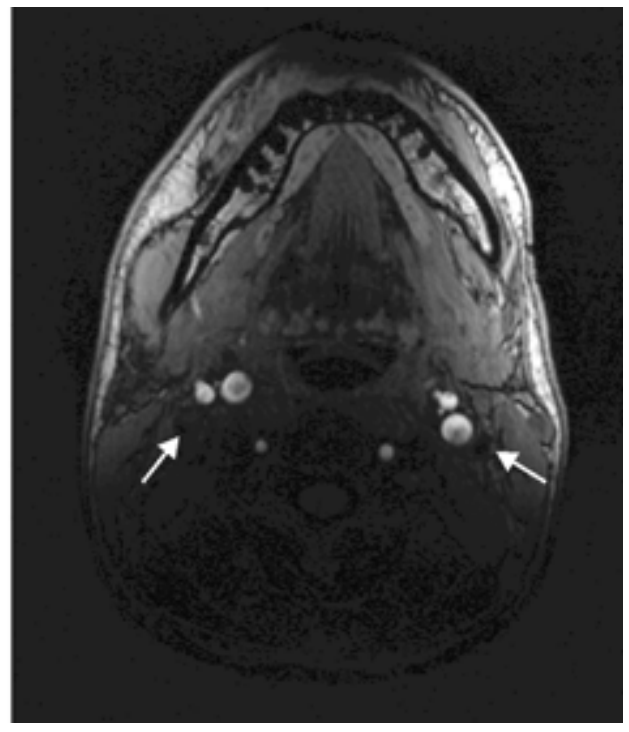

$\mathrm{D}$

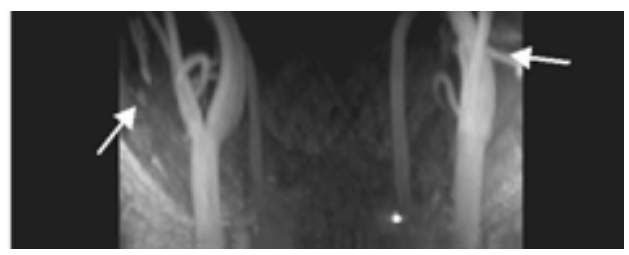

Figure B7.3.15 Transverse and coronal planes of a human neck. (A) A transverse image across the neck without a saturation pulse shows a cross-section of both the carotid artery and the jugular vein (arrows). (B) After the use of a saturation pulse, the signal from the jugular vein is dramatically suppressed. (C) and (D) are coronally reformatted images obtained the same 3-D transverse data sets used to obtain the images shown in (A) and (B), respectively. Imaging parameters: (A) to (D) $T_{\mathrm{R}} / T_{\mathrm{E}}=28 \mathrm{msec} / 6.8 \mathrm{msec}, T_{s}=10.24 \mathrm{msec}, \Delta x$ $\times \Delta y \times \Delta z=0.39 \mathrm{~mm} \times 0.78 \mathrm{~mm} \times 1.0 \mathrm{~mm}, N_{x} \times N_{y} \times N_{z}=512 \times 192 \times 70, \theta=20^{\circ}, N_{\text {acq }}=1, \tau_{R F}=1.024 \mathrm{msec}$, $G_{s s}=3.14 \mathrm{mT} / \mathrm{m}$.

A common contrast agent concentration injected in people is $0.5 \mathrm{M}$. The injection is usually given by body weight using the rule that $0.1 \mathrm{mmol} / \mathrm{kg}$ or $0.2 \mathrm{ml} / \mathrm{kg}$ should determine the dose. For a $100 \mathrm{~kg}$ person, this implies an injection of $20 \mathrm{ml}$ of the contrast agent. An important question to ask is: "What will the concentration of the contrast agent be in the blood when it has reached an equilibrium phase?" The blood volume in an adult is $\sim 5$ liters, and about half of the contrast agent is "extravasated" (i.e., it leaves the blood and is taken up elsewhere in the body) by the time the contrast agent reaches equilibrium. This implies that the concentration of the contrast agent in the blood is reduced to $1 \mathrm{mM}$ ( $0.5 \mathrm{M}$ multiplied by $20 \mathrm{ml}$ divided by 5 liters and divided by a factor of 2 due to the "extravasation" effect). For a relaxivity of $5 \mathrm{mM}^{-1} \mathrm{sec}^{-1}$ and $[c]=1 \mathrm{mM}$, the resulting $T_{1}$ of the blood $\left(T_{1, \text { post }}\right)$ will be $\sim 170 \mathrm{msec}$, assuming $T_{1, \text { pre }}=1200 \mathrm{msec}$. This will lead to bright signal from all blood for short $T_{\mathrm{R}}$ and large flip angle sequences.

\section{Suppressing signal from inflowing blood using a saturation pulse}

The nomenclature "MR angiography" is usually used for imaging of any vessels but often implies imaging arteries (MR arteriography would be more appropriate), whereas MR venography is used when referring to veins. There are situations where it is desirable to

Motion Artifacts, Motion Compensation, and Magnetic Resonance Angiography

\section{B7.3.15}


A

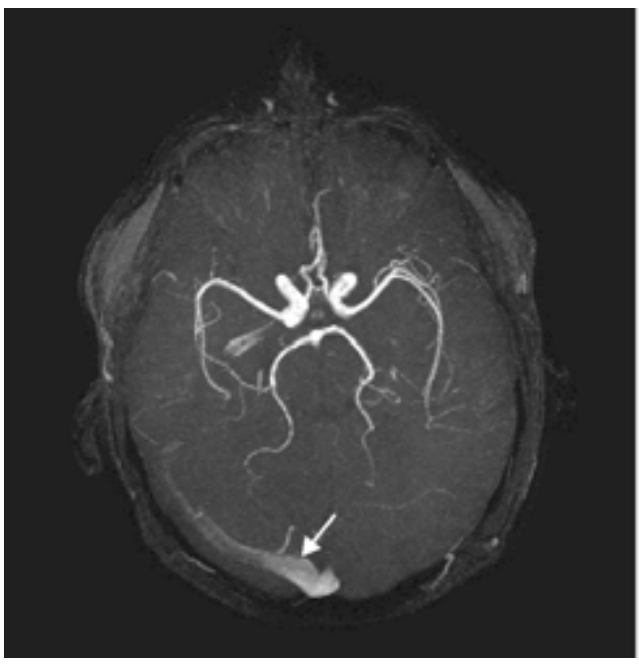

C

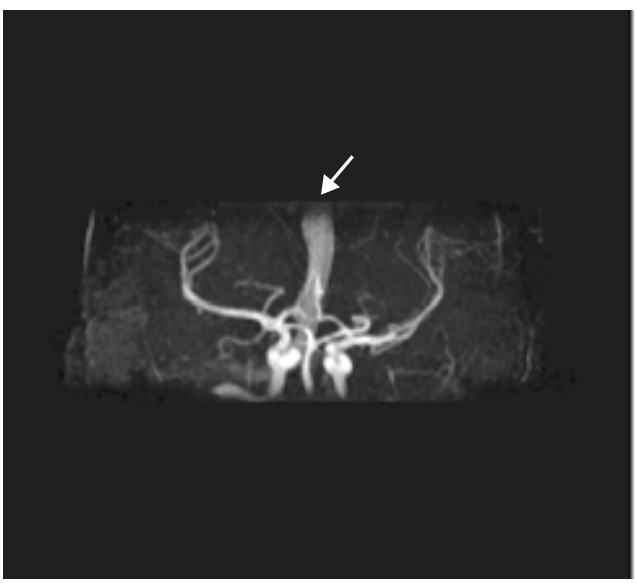

B

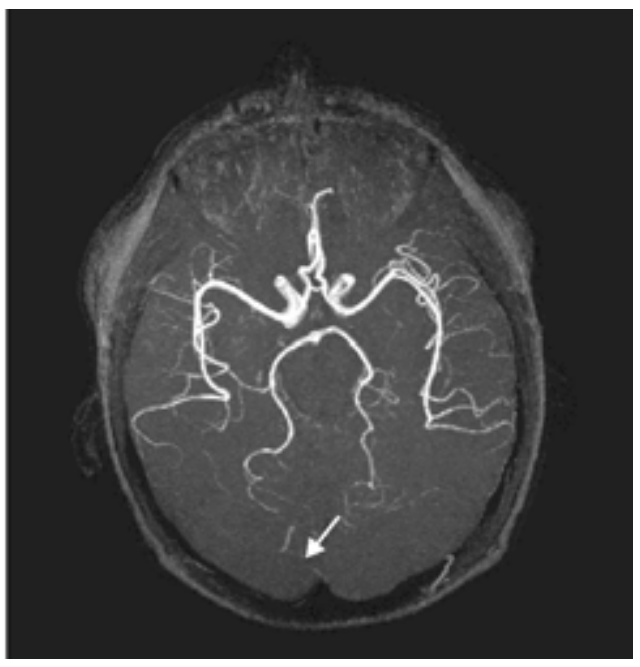

D

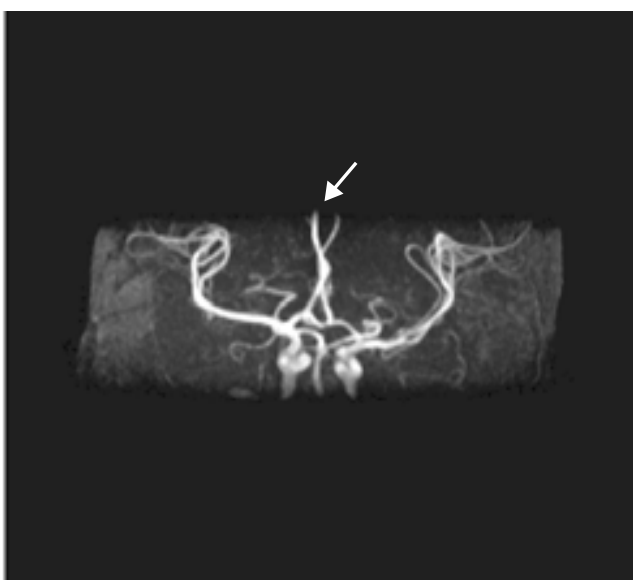

Figure B7.3.16 Comparison between MIP images showing both arteries and veins-(A) and (C)-in the brain and an MR arteriogram-both (B) and (D) -obtained by saturating the venous flow by placing a saturation pulse above the imaging slice (acquired as a transverse 3-D slab). (A) and (B) show the comparison in a transverse view while $(C)$ and $(D)$ show the coronal views from the same two data sets, respectively. The transverse sinus-arrows in $(A)$ and $(C)$ - has been suppressed successfully in both (B) and (D). Imaging parameters: (A), (C) $T_{\mathrm{R}} / T_{\mathrm{E}}=25 \mathrm{msec} / 6.8 \mathrm{msec}, T_{s}=10.24 \mathrm{msec}, \Delta x \times \Delta y \times \Delta z=0.5 \mathrm{~mm}$ $\times 1.0 \mathrm{~mm} \times 1.0 \mathrm{~mm}, N_{x} \times N_{y} \times N_{z}=512 \times 192 \times 70, \theta=15^{\circ}$, with no venous saturation pulse, $N_{\text {acq }}=1, \tau_{R F}$ $=1.024 \mathrm{msec}, G_{s s}=3.14 \mathrm{mT} / \mathrm{m}$; (B), (D) $T_{R}=28 \mathrm{msec}, \theta=15^{\circ}$, with venous saturation pulse.

Magnetic Resonance Angiography image either arteries or veins selectively. Ideally, if resolution were sufficient, separating arteries from veins in a contrast enhanced image would not be difficult. However, in practice, it is easier and more convenient to eliminate the signal from the unwanted component. This can be accomplished by using a saturation pulse upstream from the imaged slice followed shortly before the imaging RF excitation pulse (Fig. B7.3.13). The time $T_{\text {sat }}$ between the saturation and imaging pulse is determined by the velocity of the flowing spins and imaging parameters. When imaging the carotid arteries, for example, the jugular vein signal is minimized by applying a saturation pulse cranial to the excited slice because venous flow is up-to-down (Fig. B7.3.14). (The top-to-bottom direction is 
A

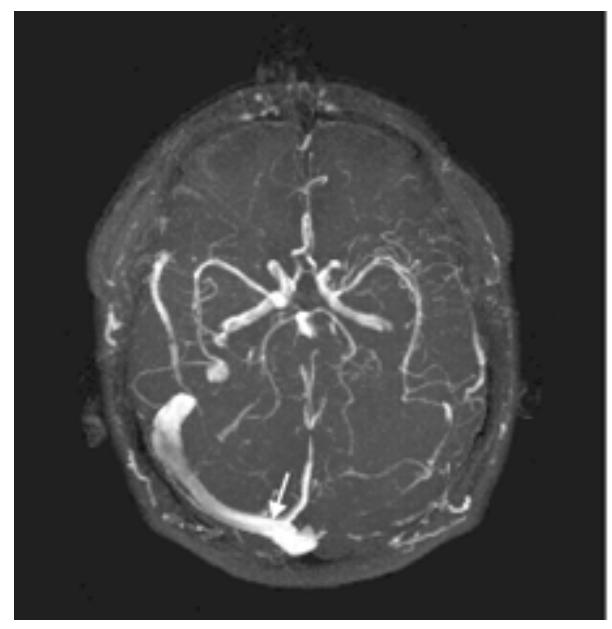

B

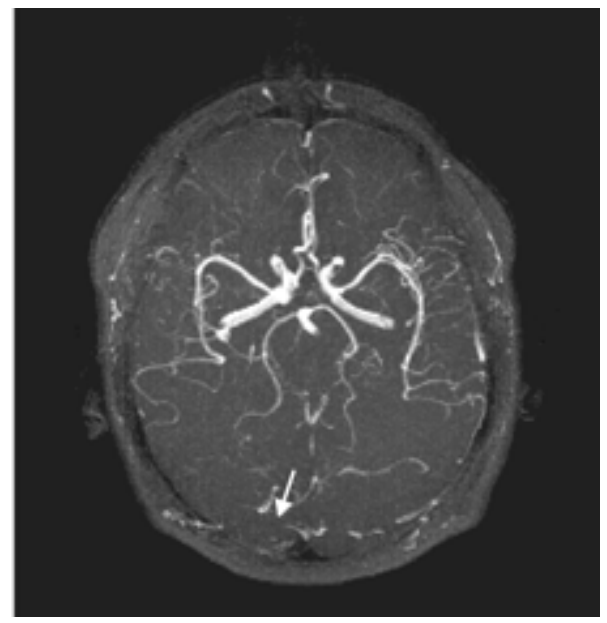

Figure B7.3.17 Comparison between MIP images obtained (A) without and (B) with the use of a venous saturation pulse obtained from a 2-D multi-slice data set with the use of saturation pulse applied before each new 2-D slice acquisition. Note the better appearance of small vessels with very slow flow in comparison with the thick slice 3-D data sets shown in Figure B7.3.16. This is because even slow flow is enhanced in the 2-D imaging case in view of the thinner slices. Note that the transverse sinus (arrow) has been suppressed in (B). Imaging parameters: (A),(B) $T_{R} / T_{E}=30 \mathrm{msec} / 9 \mathrm{msec}, T_{s}=5.12 \mathrm{msec}, \Delta x$ $\times \Delta y \times \Delta z=1.0 \mathrm{~mm} \times 1.0 \mathrm{~mm} \times 3.0 \mathrm{~mm}, N_{x} \times N_{y} \times N_{z}=256 \times 192 \times 36, \theta=35^{\circ}, N_{\mathrm{acq}}=1, \tau_{R F}=2.048$ $\mathrm{msec}, G_{s s}=8.0 \mathrm{mT} / \mathrm{m}$.

also referred to as head-to-foot or cranio-caudal.) The time between the saturation pulse and the signal generating pulse ( $T_{\text {sat }}$ in Fig. B7.3.13) is so short that the signal from the veins has no time to regrow and remains essentially zero relative to the surrounding tissue. As the blood flows into the slab in a time $T_{\mathrm{R}}$ it is again suppressed along with the other tissues and appears dark due to its long $T_{1}$ recovery. This capability is demonstrated in Figure B7.3.15. Figure B7.3.16 shows a 3-D imaging example while a 2-D MRA example is shown in Figure B7.3.17. In both cases, the venous blood signal contribution was reduced by selectively saturating the venous blood. This selective saturation method is often necessary for 2-D TOF MRA because both veins and arteries will otherwise appear bright. As a closing note on the use of saturation pulses, the reader should be aware that the more RF pulses that are applied the larger the RF power deposition (see UNIT B2.3).

\section{KEY REFERENCES}

Anderson, C.M., Edelman, R.R., and Turski, P.A. (eds.) 1993. Clinical Magnetic Resonance Angiography. Raven Press, New York.

This reference reviews the basic concepts behind time-of-flight and phase contrast imaging as well as numerous aspects of MR angiography.

Edelman, R.R., Ahn, S.S., Chien, D., Li, W., Goldmann, A., Mantello, M., Kramer, J., and Kleefield, J. 1992. Improved time-of-flight MR angiography of the brain with magnetization transfer contrast. Radiology 184:395.

This paper applied the use of magnetization transfer contrast to MRA.

Gao, J.H., Holland, S.K., and Gore, J.C. 1988. Nuclear magnetic resonance signal from flowing nuclei in rapid imaging using gradient echoes. Med. Phys. 15:809.

Introductory discussions of blood saturation during 2-D and 3-D time-of-flight appear in this article.

Motion Artifacts, Motion Compensation, and Magnetic Resonance Angiography

B7.3.17 
Haacke, E.M., Brown, R.W., Thompson, M.R., and Venkatesan, R., 1999. Magnetic Resonance Imaging: Physical Principles and Sequence Design. John Wiley \& Sons, New York.

This paper covers the technical aspects presented here, but in more detail, and also discusses more advanced materials.

Haacke, E.M., Masaryk, T.J., Weilopolski, P.A., Zypman, F.R., Tkach, J.A., Amartur, S., Mitchell, J., Clampitt, M., and Paschal, C. 1990. Optimizing blood vessel contrast in fast three-dimensional MRI. Magn. Reson. Med. 14:202.

Introductory discussions of blood saturation during 2-D and 3-D time-of-flight appear in this paper.

Lin, W., Haacke, E.M., Smith, A.S., and Clampitt, M.E. 1992. Gadolinium-enhanced high resolution MR angiography with adaptive vessel tracking: preliminary results in intracranial circulation. J. Magn. Reson. Imaging 2:277.

This paper proposed the ability to use contrast agents to enhance vessel visibility.

Marchal, G., Bosmans, H., Van Hecke, P., Jiang, Y.B., Aerts, P., and Bauer, H. 1991. Experimental Gd-DTPA polylysine enhanced MR angiography: sequence optimization. J. Comput. Assist. Tomogr. 15:711.

This article proposed the ability to use contrast agents to enhance vessel visibility.

Pike, G.B., Hu, B.S., Glover, G.H., and Enzmann, D.R. 1992. Magnetization transfer time-of-flight magnetic resonance angiography. Magn. Reson. Med. 25:372.

This article applied the use of magnetization transfer contrast to MRA.

Potchen, E.J., Haacke, E.M., Siebert, J.E., and Gottschalk, A. (eds.) 1993. Magnetic Resonance Angiography: Concepts \& Applications. Mosby-Year Book, St. Louis.

This book reviews the basic concepts behind time-of-flight and phase contrast imaging as well as numerous aspects of MR angiography.

Prince, M.R., Yucel, E.K., Kaufman, J.A., Harrison, D.C., and Geller, S.C. 1993. Dynamic gadolinium-enhanced three-dimensional abdominal MR arteriography. J. Magn. Reson. Imaging 3:877.

This text proposed the ability to use contrast agents to enhance vessel visibility.

Wolff, S.D. and Balaban, R.S. 1989. Magnetization transfer contrast (MTC) and tissue water proton relaxation in vivo. Magn. Reson. Med. 10:135.

This paper proposed the use of magnetization transfer contrast to suppress background tissue.

Contributed by Yu-Chung Norman Cheng

Case Western Reserve University

Cleveland, Ohio

E. Mark Haacke

The MRI Institute for Biomedical Research

Detroit, Michigan 